\title{
Development of coaxial speaker-like non-contact electrostatic sensor for aviation engine exhaust electrostatic character research
}

\author{
Zhaoheng Du ${ }^{182, a}$ Xiaofeng $\mathrm{Hu}^{1}$ Ming Wei ${ }^{1}$ Lei Wang ${ }^{1}$ \\ 1 Research Institute of Electrostatic and Electromagnetic Protection, Mechanical Engineering College in Shijiazhuang, China \\ ${ }^{2}$ Northwest Institute of Nuclear Technology in Xi'an, China
}

\begin{abstract}
Electrostatic sensor is the most important equipment in aero-engine exhaust electrostatic character research. By comparing a variety of sensor test programs, the coaxial speaker-like noncontact electrostatic sensor program is proposed. Numerical simulation analysis indicates the electric field distribution of electrostatic sensor, the influence principle of gap width, outer diameter, center diameter, angle and other factors on the sensor capacitance values which identify the key indicators of electrostatic sensor. The experiment test shows that the simulation analysis is in good agreement with the experimental results.
\end{abstract}

\section{Introduction}

Aero-engine exhaust electrostatic will charge aircraft to very high potential which causes a lot of electromagnetic interference to navigation system and communication system $^{[1]}$. So, it is very important to research the electrostatic character of aero-engine exhaust and its electrification effect. Electrostatic sensor is the most important equipment of aero-engine exhaust electrostatic character research $^{[2]}$.

In order to measure the electrostatic character of aeroengine exhaust, it seems a easy approach: Insulate the engine from the earth, drive the engine to work in different conditions, test the charging current between the engine and the earth directly or the amount of electrostatic charge on aero-engine directly. However, due to the huge and complex of aviation engine test platform, the basic conditions of aviation engine test platform can not meet the test requirement ${ }^{[3]}$. So, an alteration is prerequisite. According to the special requirements of electrostatic testing, the leak resistance of aviation engine test platform should be $1012 \Omega$ or bigger. It requires all the leak resistance of aviation engine test platform should be $1012 \Omega$ or bigger. In fact, the quality of such a big aviation engine test platform is so great that the resistance of insulating material which having a high volume resistivity will drop rapidly and is difficult to maintain $1012 \Omega$ or bigger. It is, actually, impossible to insulate all the part of aero-engine test platform from the earth with a resistance larger than $1012 \Omega$. Therefore, many consideration suggests that the easy approach is not suitable for the electrostatic character research of jet aircraft engines. And electrification is can not be measured directly ${ }^{[4,5]}$.

\section{Principle of electrostatic sensor influence factors of coaxial speaker-like non-contact electrostatic sensor}

According to the principle of conservation of charge, if aero-engine's working produces exhaust electrostatic electrification, there is bound to be a positive and negative charge separation and transfer. If the aeroengine carries some kind polarity charge, the aero-engine exhaust is bound to carry the opposite polarity charge. So if the exhaust charged state is measured, the electrical conditions of aircraft engines are naturally clear. Although the engine combustion chamber is in high temperature and pressure, and the physical and chemical changes are complex, the temperature of the tail nozzle is lower, the pressure is relatively smaller, the overall charge state is relatively stable, which make the exhaust can be considered as a stable gas-solid two-phase flow. High-speed charged aero-engine exhaust can be approximately considered as uniformly charged cylinder. Directional movement of positive and negative charge of aero-engine exhaust airflow forms current. And the current can be measured with the help of Rogowski coil type sensor. The current at the center of Rogowski coil type sensor generates magnetic field, and the output signal of Rogowski coil is the differential of the current. When the engine operating condition is stable, the current is kept constant or relatively slow change, so the sensor output signal is very small which make it not conducive to the test and has a poor dynamic performance. On the other hand, the Rogowski coil type sensor has a complex structure, costly and long design cycles. Therefore, it is not suitable for aero-engine exhaust electrostatic character research.

\footnotetext{
$\overline{{ }^{a}}$ Zhaoheng Du: duzhaoheng188@163.com
} 
For the special requirements of the aero-engine exhaust electrostatic character research, a sensor with high sensitive area and output signal is required to be designed. After comprehensive consideration, the electrostatic sensor designed to be non-contact coaxial speaker-like (Fig. 1). The electrostatic sensor is installed behind the engine nozzle, and is coaxial with the aeroengine exhaust airflow. Taking into account the rapid spread of the engine jet, the sensor is designed to be speaker-like in the actual test in order to prevent the damage caused by the spread of the aero-engine exhaust airflow.

The outer cylinder of the electrostatic sensor is connected to the ground and its length is bigger than the inner cylinder, so that the electrostatic sensor can be considered as an ideal Faraday cylinder. At the same time, the inner cylinder gets a good electrostatic shielding. The inner cylinder is the test electrode, and is directly connected with the electrostatic test apparatus. The inner cylinder is separated from the outer cylinder by a certain number of special electrostatic insulation support rod. The static insulation support rod adopts a multilayer composite structure, which not only ensures the reliability of internal and external cylinder connections, but also ensures the high resistance at high temperature. This coaxial speaker-like non-contact electrostatic sensor not only has high stability and sensitivity, but also has the advantages of simple structure, compact and convenient assembly and disassembly. And it will not damage the original engine system. In order to simplify the analysis of aero-engine exhaust's electrostatic electricity,

In order to simplify the analysis and discussion of the influence of aero-engine exhaust's electrostatic charge on the output voltage of the engine, the aero-engine exhaust is reduced to an uniformly charged finite length cylinder with a radius and a half lengths are Pr (meters) and Pl (in meters), which is shown at Table 1.

Table 1. Main parameters of coaxial speaker-like non-contact electrostatic sensor

\begin{tabular}{|ccccccc|}
\hline Parameters & d_in & dis & Lin & LL1 & LL2 & zeta \\
\hline Value & $0.6 \mathrm{~m}$ & $0.1 \mathrm{~m}$ & $0.5 \mathrm{~m}$ & $0.5 \mathrm{~m}$ & $0.5 \mathrm{~m}$ & $11^{\circ}$ \\
\hline
\end{tabular}



Figure1.Diagram of coaxial speaker-like non-contact electrostatic sensor.

The main design parameters are as follow: Central radius (d_in, meters), spacing between the inner and outer cylinder (dis, meters), the inner cylinder half-chord (Lin, meters), the outer cylinder on the left side chord length (LL1, meters), the outer on the right cylinder chord (LL2, meters) and wall angle with the central axis (zeta, angle degrees) equal to the inner and outer tube.

\section{Influence factors of coaxial speaker- like electrostatic sensor}

The output voltage of the coaxial speaker-like noncontact electrostatic sensor is analyzed by the finite element analysis. Simulation results with different mesh grid settings are shown in Figure 2.Among numerical calculation, the point charge is equivalent to a charged conductor sphere with radius of $\mathrm{CBr}(\mathrm{m})$ in order to simulate conditions under point charge sensor output voltage.

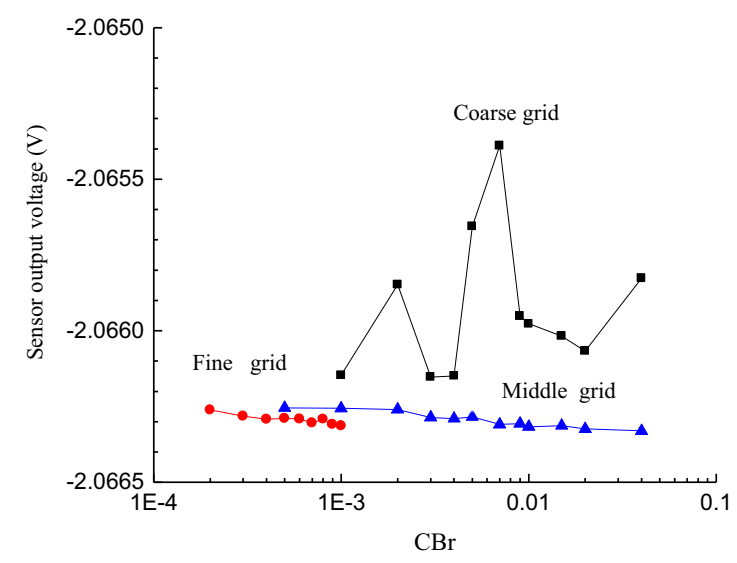

Figure 2.Sensor output voltage changes with the radius of the conductor $\mathrm{CBr}$

By comparing the results of three kinds of grid step size, it can be found that the results of the coarse grid computing are slightly greater than that of the fine grid and the middle grid. This is due to the poor accuracy of the coarse mesh, and the voltage drop of small conductor near the sphere is larger. Because the potential drop occurs mainly in the space region near the conductor sphere, and the distance from the conductor sphere is smaller, the output voltage of the sensor is smaller. So, the middle mesh grid is a better setup for numerical calculation.

The radius of the internal and external cylinder of the sensor and the distance between them are an important factor affecting the output voltage of the sensor. When the outer wall of the LL1 and the outer wall of the right size LL2 have the same value, the dis changes as 0.05 , $0.1,0.2,0.25$ respectively. The sensor output voltage with different gap width changes with the outer wall size(Fig.3 ). From Fig.3, the output voltage of the sensor decreases with the increase of the length of the outer wall, and decreases with the increase of LL1. When the LL1 reaches $1 \mathrm{~m}$, its increase will not lead to a significant decrease of the output voltage of the sensor. This shows that although the external wall length of the sensor can be improved, the anti-interference ability of the sensor can be improved, but the increase of the external wall size will result in the increase of the internal and external wall capacitance. 


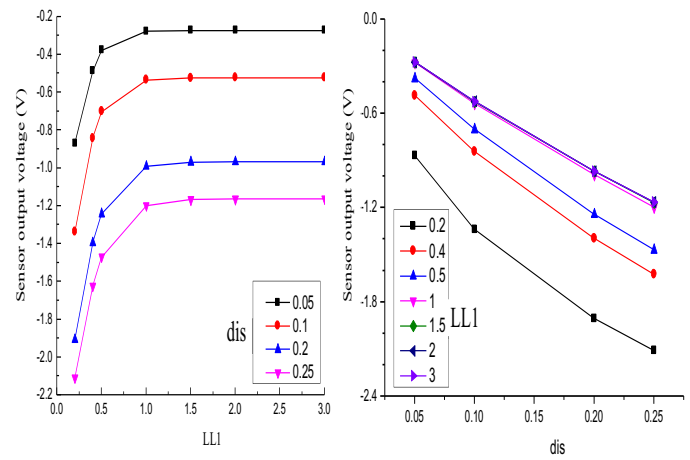

Figure3.The sensor output voltage with different dis and LL1.

As one of the key parameters of the coaxial speaker-like non-contact electrostatic sensor, the variation of the capacitance value of the capacitance value of the static sensor with the distance between different medium and different diameters is shown in Fig. 4. By the increase of the inner and outer cylinder spacing, the sensor capacitance can be reduced. The increase of the diameter will lead to the increase of capacitance, which is not difficult to explain with the law of the ideal plate capacitor.
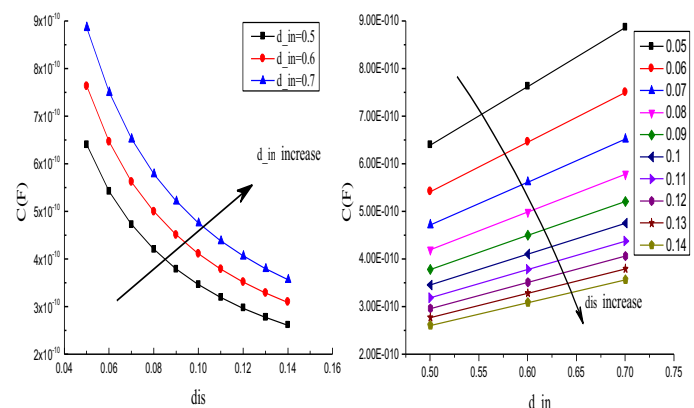

Figure 4. The sensor output voltage with different dis and d_in.

Under different spacing and angle, the capacitance value of the non-contact type coaxial horn-shaped electrostatic sensor shown in Figure 5 below. As suggested by the Fig.5, when the angle value are between 9 13 degrees, the sensor capacitance value is almost not affected by the change of the angle, the basic remain constant. The effect of the angle can be found in the range of a larger variation range, with the increase of the angle, the capacitance value of the sensor is only slightly increased, but the increase range is no more than $1 \%$. The influence of the change of the internal and external wall spacing on the capacitance value is negligible.
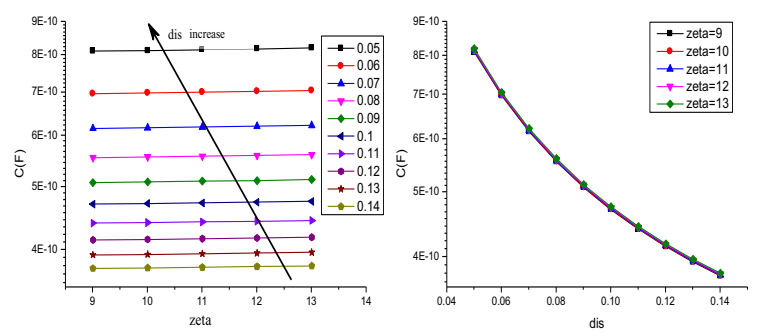

Figure 5.The sensor output voltage with different zeta and dis..

\section{Discussions and conclusions}

The results of the coarse grid computing are slightly greater than that of the fine grid and the middle grid. This is due to the poor accuracy of the coarse mesh, and the voltage drop of small conductor near the sphere is larger. Because the potential drop occurs mainly in the space region near the conductor sphere, and the distance from the conductor sphere is smaller, the output voltage of the sensor is smaller. So, the middle mesh grid is a better setup for numerical calculation.

The output voltage of the sensor decreases with the increase of the length of the outer wall, and decreases with the increase of LL1. When the LL1 reaches $1 \mathrm{~m}$, its increase will not lead to a significant decrease of the output voltage of the sensor. This shows that although the external wall length of the sensor can be improved, the anti-interference ability of the sensor can be improved, but the increase of the external wall size will result in the increase of the internal and external wall capacitance.

By the increase of the inner and outer cylinder spacing, the sensor capacitance can be reduced. The increase of the diameter will lead to the increase of capacitance, which is not difficult to explain with the law of the ideal plate capacitor.

When the angle value are between 9 13 degrees, the sensor capacitance value is almost not affected by the change of the angle, the basic remain constant. The effect of the angle can be found in the range of a larger variation range, with the increase of the angle, the capacitance value of the sensor is only slightly increased, but the increase range is no more than $1 \%$. The influence of the change of the internal and external wall spacing on the capacitance value is negligible.

\section{References}

1. Liu, S., et al, (2014) Gaodianya Jishu/High Voltage Engineering. Mechanism analysis and experimental research of aero-engine exhaust electrostatic charging, 40(9), (2014)

2. Du, Z., et al, Gaodianya Jishu/High Voltage Engineering. Modeling and simulation analysis of aerial vehicle charging and discharging process, 40(9), (2014)

3. Fu, Y., et al, Transactions of Nanjing University of Aeronautics and Astronautics. Gas path elec-trostatic sensor monitoring and comparison experiment on turbojet engine, 30(4),(2013). 
4. Du, Z., S. Liu and W. Lei,(2010) Selection of the optimal wavelet bases for wavelet de-noising of partial discharge signal. In: International Conference on Signal Processing Systems, ICSPS 2010, 5-7 July 2010,Dalian, China: IEEE Computer Society. (2010).

5. Liu, P., H. Zuo and J. Sun, (2014)IEEE Sensors Journal. The electrostatic sensor applied to the online monitoring experiments of combustor carbon deposition fault in aero-engine, 14(3), (2014). 